\title{
Stability analysis of electrical powered wheelchair-mounted robotic- assisted transfer device
}

\author{
Hongwu Wang, PhD; ${ }^{1 *}$ Chung-Ying Tsai, MS; ${ }^{1}$ Hervens Jeannis, MS; ${ }^{1}$ Cheng-Shiu Chung, MS; ${ }^{1}$ Annmarie \\ Kelleher, MS; ${ }^{1-2}$ Garrett G. Grindle, MS; ${ }^{1}$ Rory A. Cooper, $\mathbf{P h D}^{\mathbf{1}}$ \\ ${ }^{1}$ Human Engineering Research Laboratories, Department of Veterans Affairs Pittsburgh Healthcare System, Pitts- \\ burgh, PA; and Department of Rehabilitation Science and Technology, University of Pittsburgh, Pittsburgh, PA; \\ ${ }^{2}$ Center for Assistive Technology, University of Pittsburgh, Pittsburgh, PA
}

\begin{abstract}
The ability of people with disabilities to live in their homes and communities with maximal independence often hinges, at least in part, on their ability to transfer or be transferred by an assistant. Because of limited resources and the expense of personal care, robotic transfer assistance devices will likely be in great demand. An easy-to-use system for assisting with transfers, attachable to electrical powered wheelchairs (EPWs) and readily transportable, could have a significant positive effect on the quality of life of people with disabilities. We investigated the stability of our newly developed Strong Arm, which is attached and integrated with an EPW to assist with transfers. The stability of the system was analyzed and verified by experiments applying different loads and using different system configurations. The model predicted the distributions of the system's center of mass very well compared with the experimental results. When real transfers were conducted with 50 and $75 \mathrm{~kg}$ loads and an $83.25 \mathrm{~kg}$ dummy, the current Strong Arm could transfer all weights safely without tip-over. Our modeling accurately predicts the stability of the system and is suitable for developing better control algorithms to enhance the safety of the device.
\end{abstract}

Key words: center of mass, center of mass distribution, electrical powered wheelchair, model, robotic transfer device, safety, stability, Strong Arm, tip-over stability, transfer.

\section{INTRODUCTION}

When wheelchair users are transferred by a human assistant, there are risks of falling and injury to both the user and caregiver, especially over the long-term [1]. Of the 770 reported wheelchair-related accidents between 1973 and 1987, 8.1 percent occurred during transfers [2]. Of the 36,000 wheelchair-related accidents between 1986 and 1990, 17 percent were caused by transfers [3]. One in two nonambulatory patients falls to the floor and becomes injured when being transferred from a bed to a wheelchair [4]. There were more than 1,325,000 home care workers or clinicians in the United States in 2004, and this number is expected to grow by 56 percent from 2004 to 2014. Lower back injuries are a major health risk

Abbreviations: $\mathrm{COM}=$ center of mass, $\mathrm{EPW}=$ electrical powered wheelchair.

*Address all correspondence to Hongwu Wang, PhD; University of Pittsburgh, Department of Rehabilitation Science and Technology, Human Engineering Research Laboratories, 6425 Penn Ave, Suite 400, Pittsburgh, PA 15206; 412624-1255; fax: 412-822-3699. Email: how11@pitt.edu http://dx.doi.org/10.1682/JRRD.2013.11.0240 
among this group, and one estimate found that 10.5 percent of back injuries in the United States are associated with transferring patients [5]. One in every three nurses becomes injured from the physical exertion put forth while moving nonambulatory patients, costing their employers on average $\$ 35,000$ per injured nurse [6]. Three different techniques for human-assisted transfers have been studied, and it was recommended that further development and usage of mechanical devices should be investigated to decrease the risk of injuries [5]. Furthermore, clinicians in a long-term care facility reported that their risk for injury was significantly reduced when using mechanical transfer assistance devices [7].

A variety of devices exist for aiding with transfers. Examples of low-tech simple devices are transfer boards, mechanical hoists, and many styles of chairs that are specifically designed to help lower people in and out of bathtubs. High-tech examples include powered patient lift devices; the humanoid-like transfer assist robot [8]; and the Home Lift, Position, and Rehabilitation chair [9]. However, these existing devices are not easily transportable and are only for use in the home and clinical setting [6,10-11], and current transportable devices (e.g., transfer boards) can place considerable strain on the assistant. In addition, many living spaces (e.g., bathrooms, bedrooms) become overly cramped when trying to position a wheelchair, lifting device, and assistant together, which greatly constrains the manner in which dependent or assisted transfers can be performed and affects safety. An electrical powered wheelchair (EPW)-mounted, mobile, robotic-assisted transfer device called the Strong Arm was designed and developed based on our literature review and user studies [12]. A prototype of the Strong Arm has been fabricated. The Strong Arm has four joint segments, powered by electric actuators. It is attached to a commercially available PerMobil C500 (PerMobil Inc; Lebanon, Tennessee) EPW with a custom track system, which allows the device to be repositioned around the seat frame of the EPW. A commercially available transfer sling can be attached to the most distal segment of the Strong Arm. The Strong Arm is powered using the PerMobil C500 batteries and requires no extra power source. The Strong Arm has five powered degrees of freedom, which reduces the effort needed to move the person.

Since the Strong Arm has the potential to move patients outside of the base of support of the EPW-e.g., at its most unstable position when the user is in the sling at its extended length (Figure 1) - potential exists for the

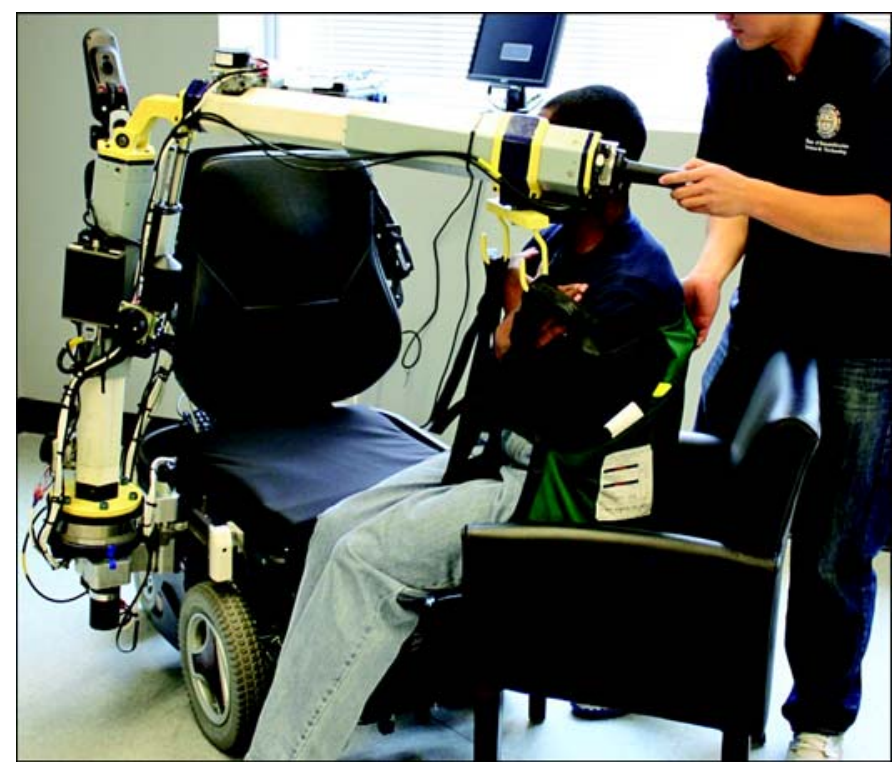

Figure 1.

Strong Arm assists with clinician transferring person in sling attached to Strong Arm from electrical powered wheelchair to other surfaces.

EPW to tip over, causing potential harm to the patient, the attendant, and the EPW. Furthermore, since the Strong Arm is designed to fit to existing EPWs, it is necessary to ensure that the device will not tip over any EPW that is suitable for its use by modeling the footprint on the EPW's base of support. We investigated the stability of the Strong Arm on a commercially available EPW with different payloads applied at various positions. The goal was to study the stability of the EPW and Strong Arm system to ensure a stable and safe operation zone for the Strong Arm based on a kinematic model of the system. Simulation and experimental results are presented to validate the model and the stability zone.

\section{DESIGN OF STRONG ARM}

The Strong Arm is a robotic arm used to assist caregivers in providing fully dependent transfers from an EPW to a bed, shower bench, toilet, or other surface. It uses three linear actuators (base segment extension, elbow flexion and extension, and forearm extension) and two rotary actuators (track movement and base segment rotation) (Figure 2). The Strong Arm mounts to the same track for positioning with respect to the seat as the assistive 


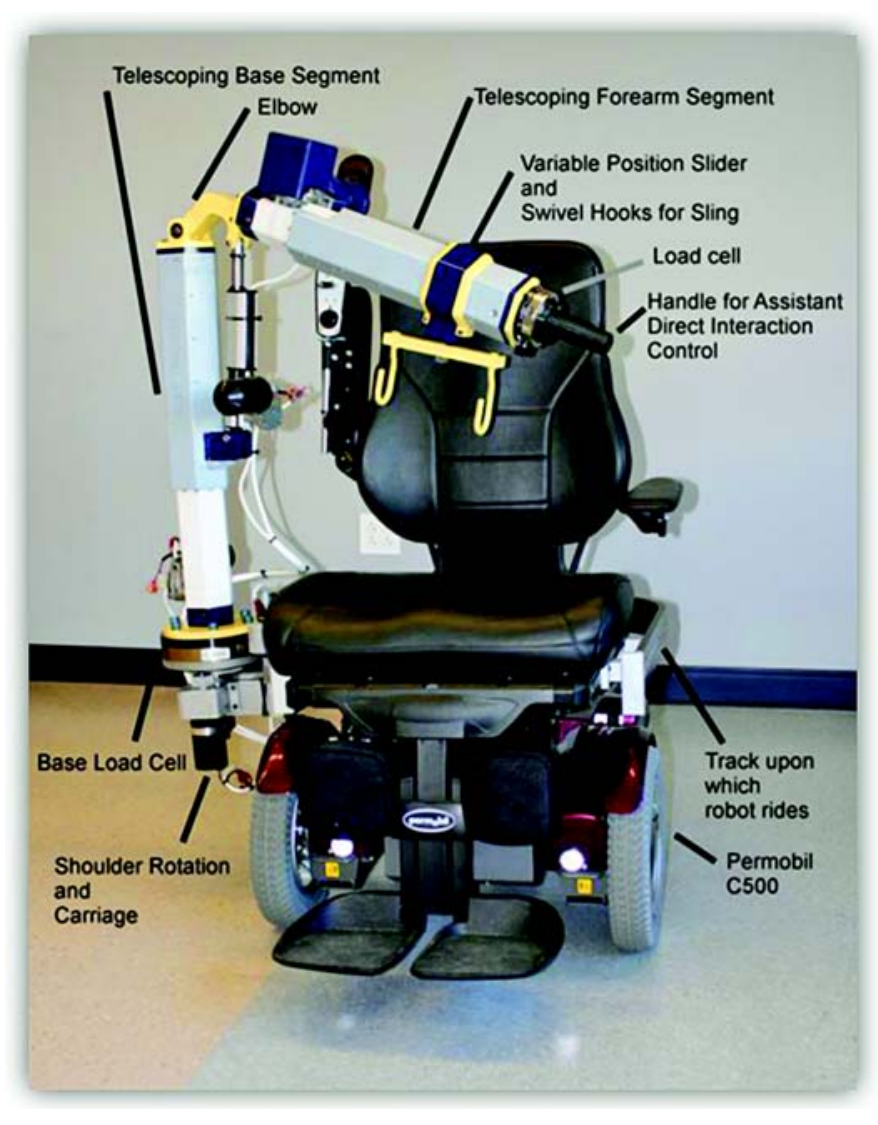

Figure 2.

Strong Arm design and attachment system for mounting on PerMobil C500 power wheelchair (PerMobil Inc; Lebanon, Tennessee).

robotic manipulators on our Personal Mobility and Manipulation Appliance [12]. The purpose of the track is to increase the robot's range of motion as well as allow the robot to be stored behind the EPW when not in use. The Strong Arm has an onboard control industrial computer with numerous buses (USB, PCI, and RS-232), which allow the device to be programmed as well as to receive input from various sensors. The ability to be programmed, along with the ability to receive information about how the system is being used (via sensors), distinguishes the Strong Arm from switch-controlled "powered” systems. The Strong Arm has encoders for highly accurate position sensing. It also has six-axis load cells to measure the forces and moments at the base and wrist. A combination of position, force, and moment data are used for direct interaction with the robot. The Strong Arm is capable of being controlled by the user, by a caregiver, or by autonomous functions. It is completely portable. The added mass of the Strong Arm, when used for 20 transfers per day, reduces the range of the PerMobil C500 by about 10 percent, which provides a more than adequate travel distance. The Strong Arm can be plugged into a wall outlet when available. The Strong Arm has four layers of safety: trained human operator, mechanical controls, electronic controls, and software controls. The mechanical layer includes shrouding of pinch points; rounded edges of metal and plastic surfaces; padding in strategic areas; and compliance, which allows the robot to elastically bend under certain loading conditions. The electronic layer includes a prominent deactivation switch, limit switches, hard force limits, hard speed limits, and user initiated emergency stops. The software layer allows for the programming of soft force limits, soft speed limits, keep-out zones, manifold constraints, and rate of loading limits. While other powered transfer devices usually include aspects of the first three layers, the software layer is unique to robotic technologies and has the potential to detect unsafe situations before they lead to accidents. These limits can be tuned or customized to individual users, which allows for maximum performance of the device without sacrificing safety.

For the current study, the Strong Arm was controlled by a direct interaction interface [13]. The handle used for direct interaction is an ergonomic plastic handle with a 4 in. grip with a diameter of 1.5 in. The handle is located at the most distal end of the robotic arm and is mounted on a multi-axis load cell used to read the forces applied to the handle. There are two multi-axis load cells (ATI Industrial Automation; Apex, North Carolina), one located at the base of the robotic arm and the other at the handle. The ATI Omega load cell at the base can hold up to $7,200 \mathrm{~N}(1,600 \mathrm{lbf})$ with a resolution of $1.5 \mathrm{~N}$ (5/16 lbf) in both $x$ - and $y$-axes. The maximum load in the $z$-axis is $18,000 \mathrm{~N}(4,000 \mathrm{lbf})$ with a resolution of $3 \mathrm{~N}$ (5/8 lbf). The ATI Delta load cell at the end effecter can achieve a reading of $660 \mathrm{~N}$ (150 lbf) with a 1/8 N (1/32 lbf) resolution in both the $x$-and $y$-axes. The maximum force on the $z$-axis is $1,980 \mathrm{~N}(450 \mathrm{lbf})$ with a resolution of $0.25 \mathrm{~N}$ (1/16 lbf). The load cell is connected to the interface power supply box, which conditions the load cell signals. Data were collected using the VxWorks real-time operating system platform (Wind River; Alameda, California) on a single-board computer Cobra EBX-12 (VersaLogic Corporation; Tualatin, Oregon). The data collected included forces and torques of both the load as well as the positions of all of the joints in the robotic arm relative to the position of the carriage. 


\section{METHODS}

\section{Kinematic Modeling of Strong Arm with Electrical Powered Wheelchair}

To develop a safe operation zone for the end user, a quasistatic stability model of the Strong Arm was created. In this model, the entire system is either stationary or moves very slowly, and thus, dynamic and inertial effects are assumed to be negligible. The payload is attached to the Strong Arm through a single point-load and was assumed not to induce nonlinear complex oscillatory behavior. The model was used to identify the basic characteristics of tipover stability of the Strong Arm and the EPW.

The tip-over point of the composite system (EPW, Strong Arm, and user being transferred) is predicted with our quasistatic model. Figure 3 illustrates the stability model of the system with single-pendulum payload. The model is composed of an EPW with two driving wheels and two caster wheels; the Strong Arm on a carriage attached to the side of the EPW; and a hook, swivel, and sling with an end-point mass. The EPW is modeled as a mass of $m_{w}$ and has a center of gravity at $l_{c o m}$ and $b_{c o m}$ away from its geometric center. The Strong Arm can be rotated through an angle $\beta$ about a point located at a distance of $l_{a}$ from the attached side of the EPW. The forearm of the Strong Arm is mounted on the top of the rotation component at a distance of $l_{a 2}$ from the EPW's geometric center. The distance from the sling and the mounting point has a length of $l_{s}$. The mass of the Strong Arm is $m_{s}$, with a center of mass (COM) at a distance of $l_{\text {scom }}$ from the rotational part. The Strong Arm can be rotated up and down through an angle $\alpha$. The EPW contacts the ground via the four wheels. The longitudinal direction distance between the driving wheels and caster wheels is $l_{w}$, and the lateral direction distance between the two driving wheels is $b_{w}$. Here, we assume the distance between the two driving wheels and caster wheels is the same. The contact forces exerted on the wheels are limited to compressive forces only. The sling and hook has a length of $l$ and a negligible mass compared with the payload $m_{p}$ attached at the hook and attached on the sling. The payload movements of the single-pendulum Strong Arm are defined in the longitudinal and the lateral directions with respect to the EPW. The angle $\varphi$ describes the payload movement in the longitudinal direction. Similarly, the angle $\theta$ describes the payload movement in the lateral direction with respect to the EPW.
In this quasistatic analysis, the entire system is assumed to be stationary or very slow moving with no payload oscillations. Therefore, the swing angles were set equal to $0^{\circ}$. In summary, the following assumptions on the tip-over stability model were made to simplify the analysis:

1. The EPW is on a horizontal flat surface.

2. The Strong Arm and EPW bodies are rigid.

3. Unspecified dimensions of the Strong Arm and EPW bodies have negligible length.

4. The payload is a point mass.

In order to evaluate the system's tip-over stability, a stability index based on the tip-over stability margin method (Force-Angle Stability Measure [14]) was introduced. This analysis method utilized the net force and couple applied to the system's COM and characterized its contribution to the tip-over moment by measuring the angle between the net force vector and the normal of each of the tip-over axes. It had a simple graphical interpretation that could be readily computed and remained sensitive to loads and applicable to general cases of uneven terrain and external disturbances. The method was selected because it did not require any integration in its computation, and in addition, it provided a useful measure of tip-over stability with minimum computation cost, making it suitable for real-time implementation on the Strong Arm.

In our Strong Arm system, it is assumed that there are four ground contact points (two driving wheels and two caster wheels), one for each tire. The COM and all external forces and moments acting on the Strong Arm, as well as the system's linear and angular accelerations, are assumed to be known. Note that all of this necessary information is either measurable or can be determined from given parameters on a real system equipped with appropriate sensory devices. We used a vision system (Vicon; Denver, Colorado) with force plates to validate the system model. From the Vicon system, we determined the distributions of COM of the system. Figure 4 shows the model for distribution of the system COM. For rotational equilibrium, the system can be expressed as Equation 1:

$$
m_{\text {com }} \vec{g} \times \overline{x_{c o m}}=m_{w} \vec{g} \times \overline{x_{w}}+m_{g} \vec{g} \times \overline{x_{g}}+m_{p} \vec{g} \times \overline{x_{p}},(1)
$$


(a)

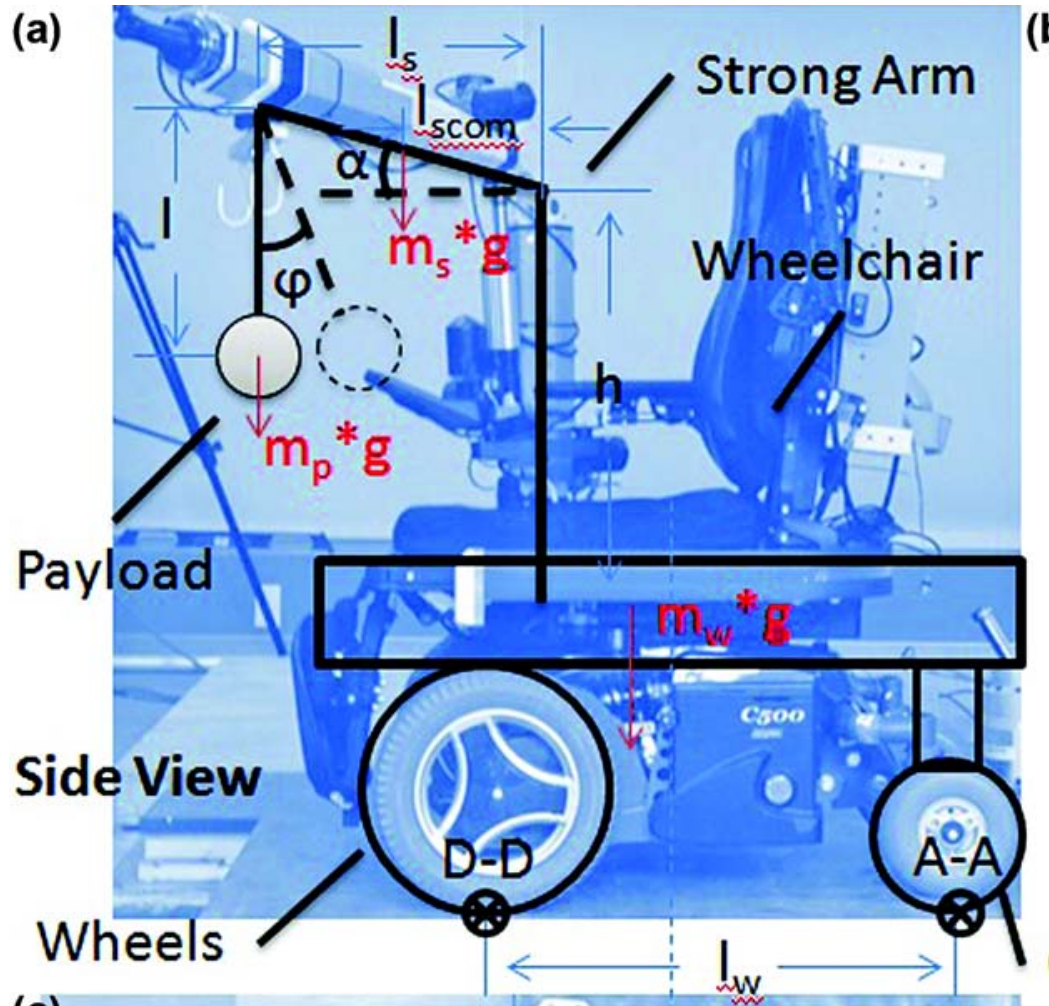

(c)

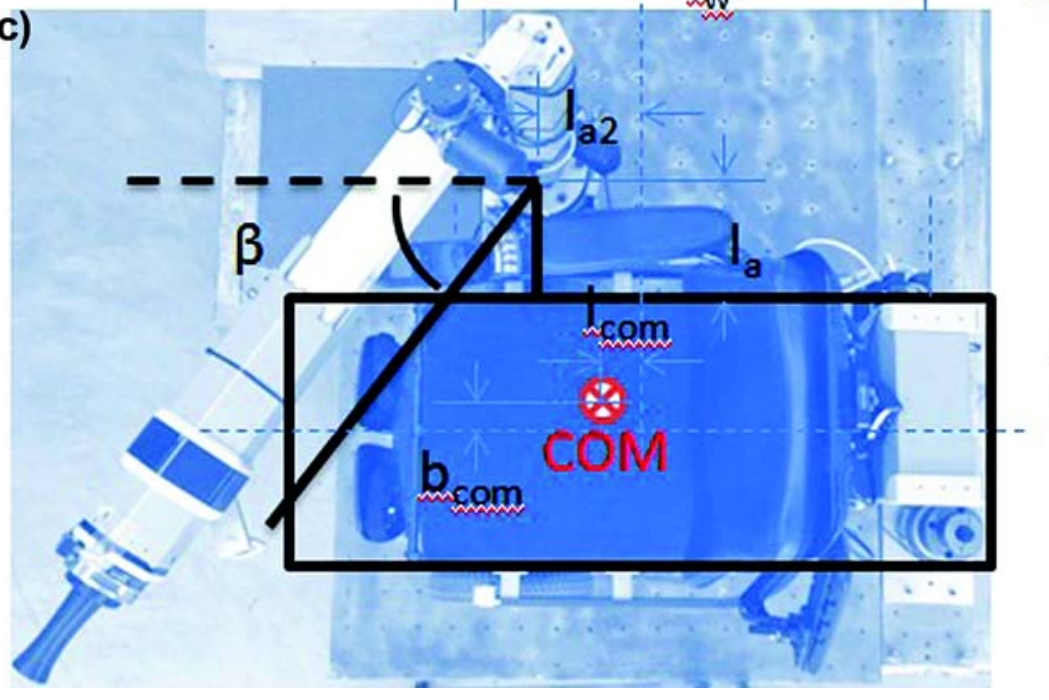

(b)

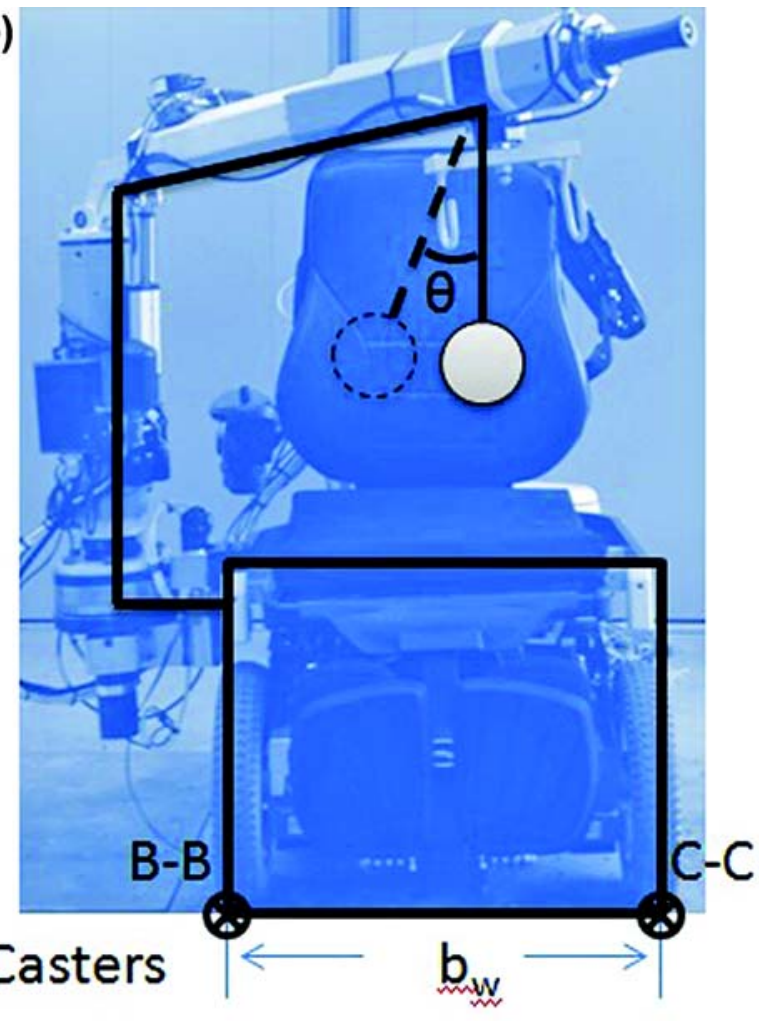

\section{FrontView}

\section{Top View}

Figure 3.

Schematic diagram of Strong Arm wheelchair system with (a) side, (b) front, and (c) top views. $\alpha=$ Strong Arm vertical rotation, $\beta=$ Strong Arm horizontal rotation, $\varphi=$ payload movement in longitudinal direction, $\theta=$ payload movement in lateral direction, A-A = back tip-over axis, B-B = side tip-over axis, $b_{c o m}=$ center of gravity away from wheelchair's geometric center, $b_{w}=$ lateral direction distance between two driving wheels, C-C = side tip-over axis, COM = center of mass, D-D = front tip-over axis, $g=$ gravity, $I=$ length of sling and hook, $l_{a}=$ distance from attached side of wheelchair, $l_{a 2}=$ distance of Strong Arm's forearm from wheelchair's geometric center, $I_{\text {com }}=$ center of gravity away from wheelchair's geometric center, $I_{s}=$ distance of Strong Arm's sling and mounting point from wheelchair's geometric center, $I_{\text {scom }}=$ distance of Strong Arm's center of mass from rotational part, $I_{w}=$ longitudinal direction distance between driving wheels and caster wheels, $m_{p}=$ payload mass, $m_{s}=$ Strong Arm mass, $m_{w}=$ wheelchair mass. 
where the vectors are described as Equation 2:

$$
\overline{x_{p}}=x_{p} \vec{l}+y_{p} \vec{j}+z_{p} \vec{k}, \vec{g}=g \vec{k} .
$$

Therefore, each component can be calculated as Equations 3 and $\mathbf{4}$ :

$$
\begin{gathered}
x_{c o m}=\frac{\left(m_{w} g\right) x_{w}+\left(m_{g} g\right) x_{g}+\left(m_{p} g\right) x_{p}}{m_{w} g+m_{g} g+m_{p} g} \text { and } \\
y_{\text {com }}=\frac{\left(m_{w} g\right) y_{w}+\left(m_{g} g\right) y_{g}+\left(m_{p} g\right) y_{p}}{m_{w} g+m_{g} g+m_{p} g} .
\end{gathered}
$$

According to Figure 4, the relationship between payload location $\left(x_{p}\right)$ and COM of the Strong Arm $\left(x_{s}\right)$ is listed as Equations 5 and 6:

$$
\begin{gathered}
x_{p}=x_{c}+l_{p} \sin \vartheta, y_{p}=y_{c}+l_{p} \cos \vartheta \text { and } \\
x_{s}=x_{c}+l_{s} \sin \vartheta, y_{s}=y_{c}+l_{s} \cos \vartheta .
\end{gathered}
$$

By substituting $x_{p}, y_{p}, x_{s}$, and $y_{s}$ in Equations 5 and $\mathbf{6}$ into Equations 3 and 4, we can describe $x_{\text {com }}$ and $y_{\text {com }}$ as functions of payload $\left(m_{p}\right)$ and Strong Arm rotational angle $(\vartheta)$ (Equations 7-8):

$$
\begin{aligned}
& x_{\text {com }}=F_{x}\left(m_{p}, \vartheta\right)=\frac{\left(m_{w} g\right) x_{w}+\left(m_{g} g\right)\left(x_{c}+l_{g} \sin \vartheta\right)+\left(m_{p} g\right)\left(x_{c}+l_{p} \sin \vartheta\right)}{m_{w} g+m_{g} g+m_{p} g} \text { and (7) } \\
& y_{\text {comm }}=F_{y}\left(m_{p}, \vartheta\right)=\frac{\left(m_{w} g\right) y_{w}+\left(m_{g} g\right)\left(y_{c}+l_{g} \cos \vartheta\right)+\left(m_{p} g\right)\left(y_{c}+l_{p} \cos \vartheta\right)}{m_{w} g+m_{g} g+m_{p} g} . \text { (8) }
\end{aligned}
$$

The prediction model was used to perform a tip-over stability analysis by calculating the stability angle margin when the EPW remains stationary, i.e., it does not tipover. Utilizing the prediction model, a static stability analysis was conducted for the entire range of Strong Arm angles and positions. For every Strong Arm position, the COM distributions of the system were computed.

\section{Experimental Verification}

To verify the accuracy of the prediction model, experimental data were collected and compared with predicted values. Figure 5 shows the setup that was used to

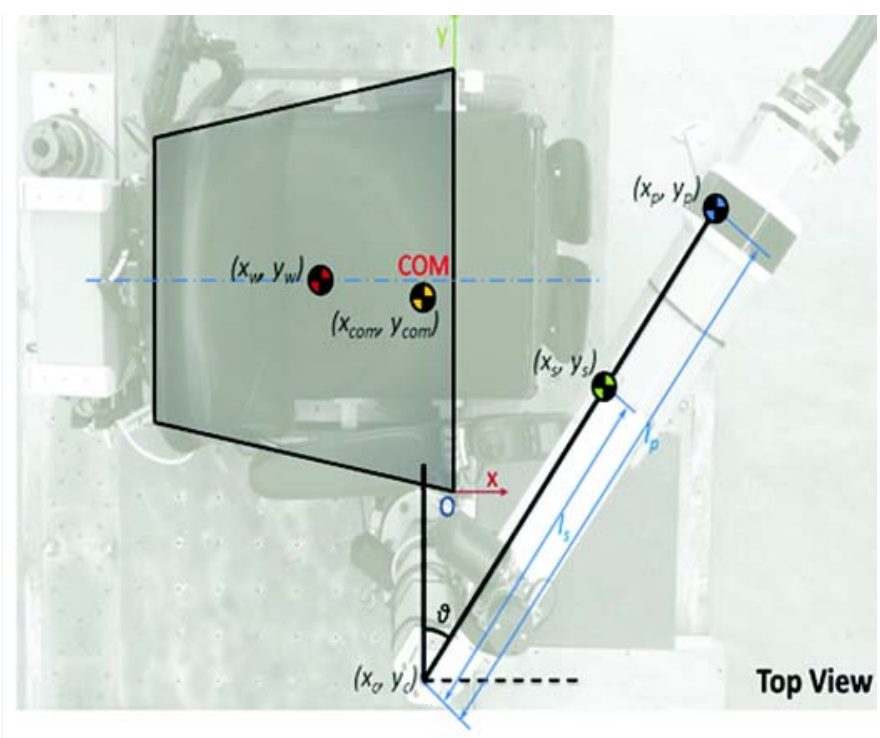

Figure 4.

System center of mass (COM) distribution with regards to Strong Arm rotation and payload. $\vartheta=$ Strong Arm rotational angle, $I_{p}=$ distance from shoulder joint to where payload is applied, $I_{s}=$ distance of Strong Arm's sling and mounting point from wheelchair's geometric center, $O=$ origin of system, $x=x$ axis, $x_{c}=x$-axis location of where Strong Arm is attached, $x_{\text {com }}=$ $x$-axis location of center of mass of system, $x_{p}=x$-axis location of where payload is applied, $x_{S}=x$-axis location of center of mass of Strong Arm, $x_{W}=x$-axis location of center of mass of wheelchair, $y_{c}=y$-axis location of where Strong Arm is attached, $y_{\text {com }}=y$-axis location of center of mass over overall system, $y_{p}=y$-axis location of where payload is applied, $y_{s}=y$ axis location of center of mass of Strong Arm, $y_{w}=y$-axis location of center of mass of wheelchair.

experimentally verify the analysis. During the test, the Strong Arm was moved to the front edge of the seat (position 1). It was positioned at the contralateral side of the surface to be transferred to. The Strong Arm position was adjusted by moving the carriage along the track on the EPW. It could be positioned by adjusting the rotational angle $\beta$ (we define $\vartheta=0^{\circ}$ [Figure 4], when the Strong Arm's forearm is aligned with the axis of two driving wheels) and up and down by either the up or down motion of the shoulder or adjusting the angle of $\alpha$. In our experiment, we looked at the maximum payload and end-point position before tip-over of the system; therefore $\alpha$ was set to $0^{\circ}$. Because of the symmetry in the setup, the experiments were performed only for the 


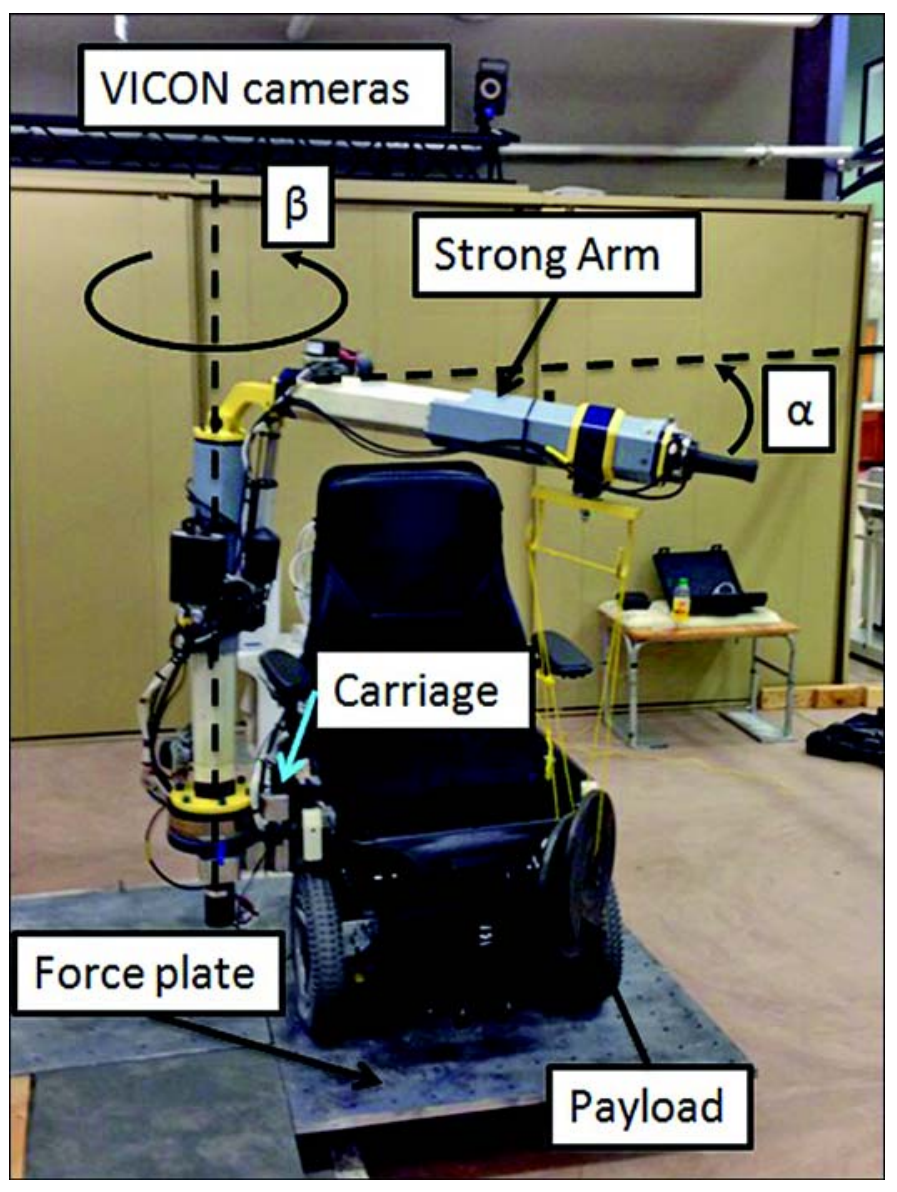

Figure 5.

Experimental setup. $\alpha=$ Strong Arm vertical rotation, $\beta=$ Strong Arm horizontal rotation.

rotational angle between $0^{\circ}$ and $180^{\circ}$. The EPW with the attached Strong Arm was positioned on a $4 \times 3 \mathrm{ft}$ force plate (Bertec Corporation; Columbus, Ohio). Reflective markers were placed on the four wheel axles of the EPW, the end of the Strong Arm elbow, the rotation center of the Strong Arm elbow, and the Strong Arm hook position (Figure 6). A 10-camera, three-dimensional motion capture system (Vicon) was used to collect the marker positions during the stability testing. Kinetic data from the force plate was collected at $1,000 \mathrm{~Hz}$. The kinematics data from all the cameras were collected at $100 \mathrm{~Hz}$.

The experimental protocol can be separated into two parts: static stability testing and dynamic stability testing. The Strong Arm's payload position can be elongated from $0.50 \mathrm{~m}$ (compact position) to $0.74 \mathrm{~m}$ (fully extended position). We tested the static stability of the system when the Strong Arm was in the compact, midpoint

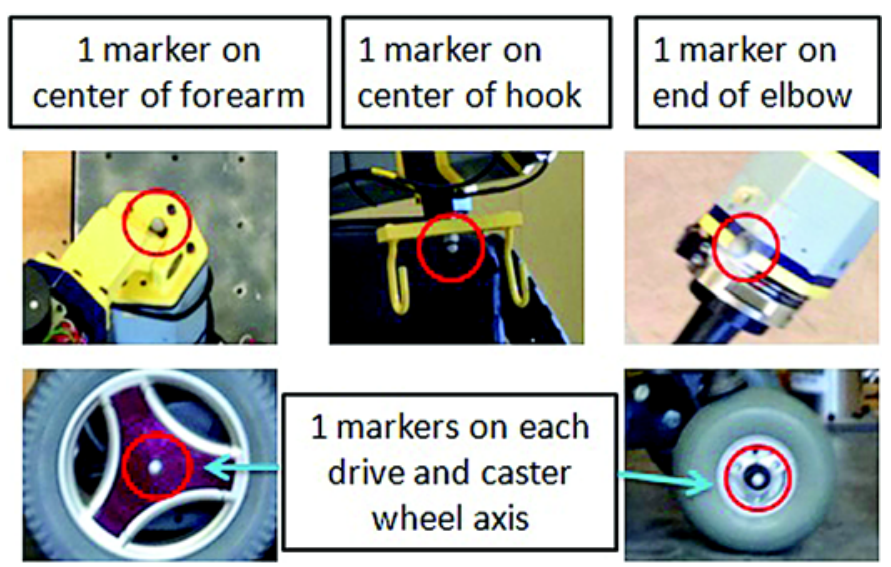

Figure 6.

Marker positions for experiments.

(0.62 $\mathrm{m})$, and fully extended positions with different payloads. The position of the Strong Arm relative to the EPW can be moved from the end of the track (near the back of the EPW seat) to $0.4 \mathrm{~m}$ forward from the back of the seat. We tested static stability in 0.1-m increments for a total of three positions. The Strong Arm's elbow can rotate $360^{\circ}$, with $0^{\circ}$ defined as the arm parallel to the axle of the front wheels and proximal to the seat. For the static stability test, we positioned the Strong Arm $180^{\circ}$ relative to the front wheel axle and tested different arm lengths (fully extended, midpoint, and compact), arm positions (three positions: at the front, $0.1 \mathrm{~m}$ backward position, and $0.2 \mathrm{~m}$ backward position), and weights (from 0-67.5 kg in $7.5 \mathrm{~kg}$ increments) to determine the COM change of the composite system.

In addition to the static tests, we conducted dynamic stability tests. In order to understand the COM change during Strong Arm operation in real situations, we looked at the COM change in the Strong Arm at full extension and swung from $0^{\circ}$ to $90^{\circ}$ in the transfer positions and orientations. For the last test, we transferred three different weights (50 and $75 \mathrm{~kg}$ and a dummy of $83.25 \mathrm{~kg}$ ) with the Strong Arm to check the performance of the model when doing real transfers. Those three weights were selected because the average weight of a National Aeronautics and Space Administration American male crewmember is $82.2 \mathrm{~kg}$ [15] and a Japanese female crewmember is $51.5 \mathrm{~kg}$ [16]. It was also reported by the National Health and Nutrition Examination Survey III [17] that the 50th percentile of body weights are close to 84 (White), 82 (Black), and $82 \mathrm{~kg}$ (Hispanic) for males 
and close to 70 (White), 80 (Black), and $72 \mathrm{~kg}$ (Hispanic) for females.

Kinetic and kinematic data were filtered using MATLAB (The MathWorks Inc; Natick, Massachusetts) with a fourth-order, zero-lag, low-pass Butterworth filter with a cutoff frequency of 5 and $7 \mathrm{~Hz}$ [18]. The model and results were analyzed using MATLAB.

\section{RESULTS}

\section{Stability Analysis}

Before applying loads to the system, we collected data to calculate the parameters for the model. The Table lists the geometric parameters and constants for the experimental setup.

After measuring and calculating the parameters for the model, the relationship between the COM of the system and the load, rotation angle, and initial position, as well as the length of where the load attached, could be expressed as Equations 9 and 10:

$$
\begin{aligned}
& x_{\text {com }}=\frac{\left[x_{c}+L \times \sin (\vartheta / 180 \times \pi) \times m_{l} \times g+W_{A} \times \sin (\vartheta / 180 \times \pi)+T_{c x}\right]}{\left(W_{T}+m_{l} \times g\right)} \text { and } \\
& y_{\text {com }}=\frac{\left[y_{c}+L \times \cos (\vartheta / 180 \times \pi) \times m_{l} \times g+W_{A} \times \cos (\vartheta / 180 \times \pi)+T_{c y}\right]}{\left(W_{T}+m_{l} \times g\right)} .
\end{aligned}
$$

\section{Static Stability Analysis of System}

Figures A and B in the Appendix (available online only) show the results of the static stability analysis. The parameters from the Table were used for calculation. To make the results easy to understand, for all of the figures the origin of the coordinate system is at the center of the right front wheel. The $y$-axis is along the axis of the two front wheels. The positive $x$-axis points forward from the back of the EPW and parallel with the longitudinal line of the EPW. The positive $y$-axis is from Strong Arm side to the opposite side of the EPW. The MATLAB plots in Appendix Figure A show the $x$ (left) and $y$ (right) positions of the center of gravity of the system when different loads from 0 to $250 \mathrm{~kg}$ were applied on the Strong Arm with the Strong Arm rotated at $0^{\circ}, 30^{\circ}, 60^{\circ}$, and $90^{\circ}$. In Appendix Figure A (left) (EPW front face up), the blue dotted line (line $y=0$ ) is the front footprint of the EPW, and in Appendix Figure A (right) (EPW front face right), the blue dotted line (line $y=-0.146 \times x$ ) is the right side footprint of the EPW. Therefore, any configurations with $x$ positions above the blue dotted line of Figure 7 (left) mean the system will tip over forward, and configurations with $y$ positions below the blue dotted line of Figure 7 (right) mean the system will tip over toward the right side. The model prediction shows that when the Strong Arm is nested in the most stable position, the composite system remains stable even with the maximum rated payload. For a $30^{\circ}$ rotation, the system can support up to $135 \mathrm{~kg}$ without tipping to the front. With the rotation angle increased from $60^{\circ}$ to $90^{\circ}$, the maximum payloads before tip-over were decreased to 70 and $60 \mathrm{~kg}$, respectively. Appendix Figure A shows that with the maximum payload $(135 \mathrm{~kg}$ ) applied, the system will not tip over to the right side.

In Appendix Figure $\mathrm{B}$, we changed the rotation angles to $90^{\circ}, 120^{\circ}, 150^{\circ}$, and $180^{\circ}$. From Appendix Figure $\mathrm{B}$, it is shown that for $90^{\circ}, 120^{\circ}$, and $150^{\circ}$, the maximum loads for preventing tip-over to the front of the EPW are 60, 70, and $135 \mathrm{~kg}$, respectively. For $180^{\circ}$, the system will tip over to the right side when the weight exceeds $100 \mathrm{~kg}$, and $125 \mathrm{~kg}$ for $150^{\circ}$. By combining results for both sides of the EPW, the maximum loads for $90^{\circ}, 120^{\circ}, 150^{\circ}$, and $180^{\circ}$ should be $60,70,125$, and $100 \mathrm{~kg}$, respectively.

Table 1.

Experimental parameters for Strong Arm and wheelchair.

\begin{tabular}{lcl}
\hline Variable & $\begin{array}{c}\text { Measured and Calculated } \\
\text { Value of Variable }\end{array}$ & Definition of Variable \\
\hline $\mathrm{g}$ & $9.81 \mathrm{~N} / \mathrm{m}^{2}$ & Gravity of earth. \\
$\mathrm{p}$ & 3.14 & Constant. \\
$\mathrm{W}_{\mathrm{T}}$ & $2,315 \mathrm{~N}$ & Measured total weight of wheelchair and Strong Arm. \\
$\mathrm{l}_{\mathrm{p}}$ & $0.74 \mathrm{~m}$ & Length of payload from rotational point when arm is fully extended. \\
$\mathrm{W}_{\mathrm{A}}$ & $64 \mathrm{~N}$ & Calculated constant torque by Strong Arm toward center of gravity of forearm. \\
$\mathrm{T}_{\mathrm{cx}}$ & $-484 \mathrm{~N}$ & Calculated constant torque by Strong Arm on $x$-axis. \\
$\mathrm{T}_{\mathrm{cy}}$ & $575 \mathrm{~N}$ & Calculated constant torque by Strong Arm on $y$-axis. \\
$\left(x_{c}, y_{c}\right)$ & $(-0.03,0.01) \mathrm{m}$ & Initial position of carriage. Most transfers will be conducted at this position. \\
\hline \hline
\end{tabular}




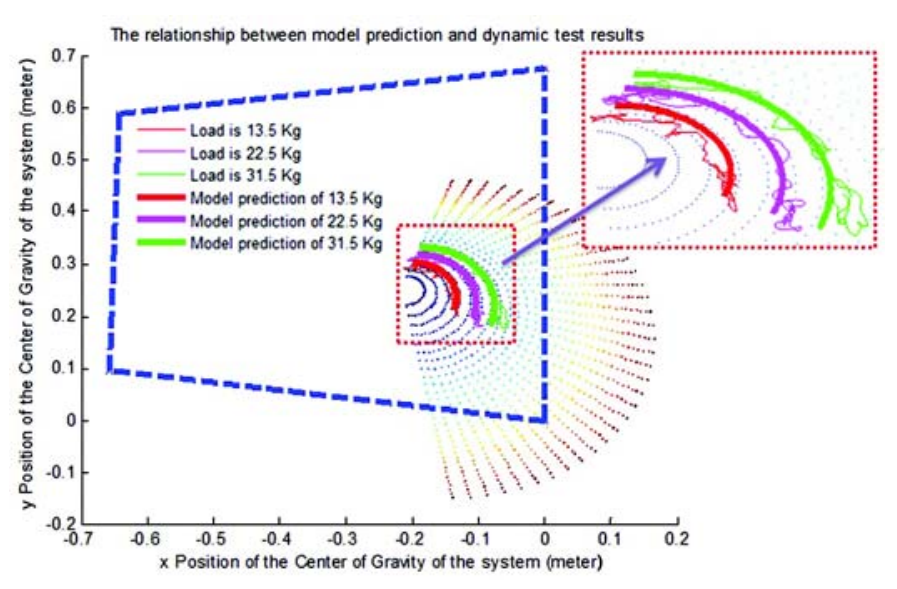

Figure 7.

Center of mass distribution when Strong Arm is fully extended at front position and operated dynamically with three different loads and model predictions.

Appendix Figure $C$ shows the relation between the maximum loads of the Strong Arm and the rotation angle when different weights of loads are applied. For loads of $50 \mathrm{~kg}$, the Strong Arm could be operated from $0^{\circ}$ to $180^{\circ}$ without tip-over to either the front or side of the EPW. When the load increases to $100 \mathrm{~kg}$, it is safe to operate the Strong Arm between $0^{\circ}$ and $41^{\circ}$ (this is enough range of movement for most transfers based on our pilot transfer trials; more data will be collected to verify this work zone). The angles decreased to $28^{\circ}$ and $20^{\circ}$ for loads of 150 and $200 \mathrm{~kg}$, respectively. Appendix Figure C shows the tip-over for side angles; 50 and $100 \mathrm{~kg}$ loads will not tip the system over to the side. For 150 and $200 \mathrm{~kg}$, the system will tip over to right side when exceeding $157^{\circ}$ and $135^{\circ}$, respectively.

From these figures in the Appendix, the model was capable of predicting when the system will tip over based on the position and orientation of the end-point of the Strong Arm with various payloads applied on the system. The figures indicate that the system becomes less stable when loads increase and has considerably less stability margin when the Strong Arm is extended to the front side of the EPW $\left(80^{\circ}-100^{\circ}\right)$.

\section{Static Stability Verification}

To verify the accuracy of the tip-over prediction model by experiment, we compared the COM distribution measured from the force plate and Vicon system with the predicted values from our model. For all the fig- ures mentioned next, we used the scatter plot function in MATLAB to display the weight distribution in circles. As the weight increased, the circles became bigger and spread out. As shown in Appendix Figure D, the blue dashed line is the EPW footprint. The circles with different colors represent COM positions of different angles for the Strong Arm during the experiment. For the $30^{\circ}$ angle, we placed $0,22.5,33.75,45$, and $54 \mathrm{~kg}$ loads on the Strong Arm. For $45^{\circ}$, we placed 11 different loads from 0 to $67.5 \mathrm{~kg}$ on the Strong Arm. For $90^{\circ}$, we tested five different loads from 0 to $25 \mathrm{~kg}$ (these values were smaller than previous weights since the system became less stable with heavier weights when rotated to $90^{\circ}$ ). From Appendix Figure D we can see that for the different angles and weights that we picked for the experiment, the model predictions of COM distributions agreed and were consistent with the real measurements from the force plate.

The prediction model was also tested at different Strong Arm positions and at different lengths of extension of the Strong Arm to test model robustness. Appendix Figure E shows the COM distribution with the Strong Arm rotated to $180^{\circ}$ with varying loads applied at three different positions (at the front, moved back $0.1 \mathrm{~m}$, and moved back $0.2 \mathrm{~m}$ ). The plot shows that our model fits the real measurements well, and at $180^{\circ}$ rotation, as the carriage moves back to the EPW, the system is more likely to tip over to the right side than the front at the same load. In addition, as the load increases, the system becomes less stable, which agrees with what we found previously. Appendix Figure F shows the COM distribution when the Strong Arm is rotated to $180^{\circ}$ with varying loads applied in the fully extended, middle, and compact positions. Again, the model predicts the real measurements well.

\section{Dynamic Stability Verification}

We tested our model's sensitivity to changes for different parameters and our model shows robustness for all parameters. Although when developing the model we did not consider dynamic effects (the movement of the loads, the inertia of the load, and the Strong Arm, etc.), we tried to compare the COM distribution when operated dynamically with our model prediction results as well. Since a real transfer happens within the $0^{\circ}$ to $90^{\circ}$ zones with Strong Arm at the front position most of time, for the dynamic test we rotated the Arm from $0^{\circ}$ to $90^{\circ}$ while at the front position. In addition, we only looked at the 
Strong Arm fully extended. The linear displacement velocity of the Strong Arm during the dynamic transfers was $0.02 \mathrm{~m} / \mathrm{s}$. The manipulator/operator was not manipulating the Strong Arm during the dynamic transfers. Figure 7 shows the COM distributions when three different loads were applied during dynamic movement of the Strong Arm and the model-predicted COM distributions of three same weight loads. Figure 7 shows that the model still predicted the COM distribution relatively well in regards to the experimental results. Due to the inertia of the loads and the Strong Arm itself, some COM distributions from the experiments were underpredicted (i.e., it is safe for our model but might not be in reality). Especially at the end of the movement when we release the arm, the large inertia causes the vibration of the COM, which leads to instability of the system (as shown in the plot). During the overall transfer process, when we operated the Strong Arm slowly, the model worked well. In addition, we did not try to hold the loads during the transfers. For real transfers, the clinicians could hold the body of the user to decrease the free user body movements. Therefore if a well-designed protocol could be developed and followed by the clinicians and users during the transfer, and the user could be as still as possible to decrease inertia, the model will work even better.

\section{Real Transfer Stability Verification}

We conducted transfers with weights comparable with human weights. For the $50 \mathrm{~kg}$ transfer, as shown in Figure 8, the model (black line) predicted the COM distributions for the three transfer trials poorly since the model indicated the COM distribution was farther from the footprint than the real COM distribution. Part of the reason was that the mounting of the Strong Arm deformed due to the heavier load (Appendix Figure G). In order to correct this, a linear compensator based on the relationship between deformations caused change of $l_{p}$ (as shown in the Table: length of the payload from the rotational point when arm fully extended) and the loads applied were added as a compensator (we estimated the maximum load of Strong Arm before breaking and used $\left.l_{p}^{\text {new }}=l_{p}+w_{\text {loads }} / w_{\text {maximum }}\right)$.

With the compensation, as shown in Figure 8 (cyan line), the model predicted the COM distribution well again. Although the measured COM distributions were not completed on the model line with compensation since the model does not take into consideration the inertia effect, it is clear that the real COM distribution is within

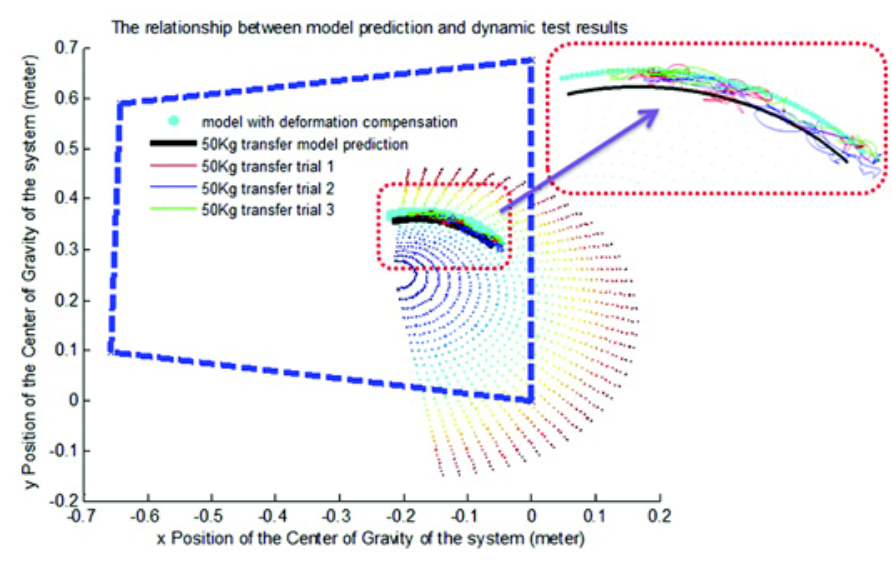

Figure 8.

Center of mass distribution when Strong Arm transfers $50 \mathrm{~kg}$ weight.

the model prediction, which means the model is actually more conservative than real measurement. This will guarantee the system without tip-over. Furthermore, the inertia effect actually decreased with $50 \mathrm{~kg}$ weight compared with Figure 8 results with lower weights, which means the model actually worked better for heavier weights.

When we transferred the $75 \mathrm{~kg}$ weight, similarly, the original model failed to predict the COM distributions for the three transfer trials (Figure 9, black line). The model did not predict the real COM distributions since the real COM distributions were closer to the edge of the footprint than the model predicted. With the compensator added, the model predicted the COM distribution well again (Figure 9, cyan line); the real COM distributions

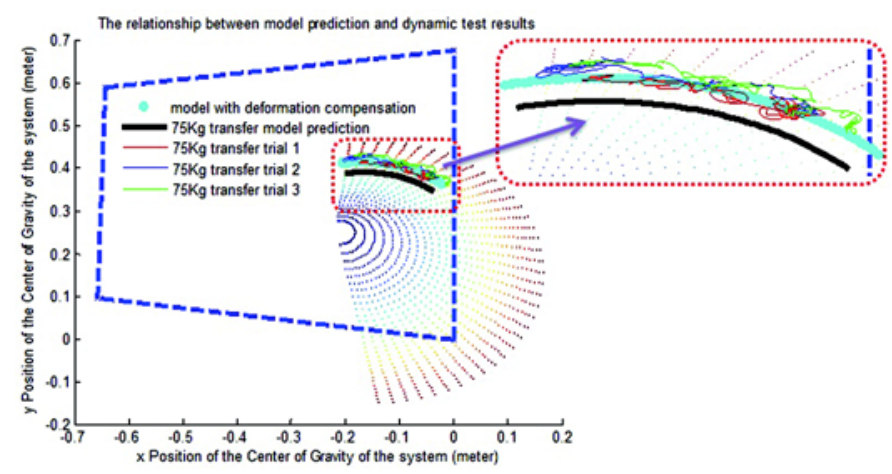

Figure 9.

Center of mass distribution when Strong Arm transfers $75 \mathrm{~kg}$ weight. 
were within the model prediction line. As shown in Figure 9, the actual inertia effect was decreased as well.

Figure 10 shows the COM distributions when the dummy was transferred. During these transfers, we used the developed transfer protocol (we started at the arm fully compact, then lifted the arm up, rotated the elbow out, and extended the elbow for positioning on the surface to be transferred) to transfer the dummy from the EPW to a toilet. As Figure 10 (left) shows, for all three transfers, the system was stable and the measured COM positions all fell within the footprint of the EPW, which means our system successfully transfers $83.25 \mathrm{~kg}$ loads without problems. In addition, from Figure 10 (right), it is clear that the model without compensation could not predict the whole transfer process correctly (when we extend the elbow, the real COM positions were out of the prediction line), but with the compensation, during the whole transfer process the real COM positions were under the safe distribution zone as predicted by the model.

\section{DISCUSSION}

Some limitations exist to the approach we presented. First, the model is based on the static stability analysis so it may not predict the dynamic stability very well. Fortunately, during the transfer, we will conduct the transfer slowly enough to keep the whole process safe. In addition, better transfer protocol and training of the use of the device could help. Second, when we created the model, both the Strong Arm and EPW were treated as rigid bodies. But in reality, there were noticeable deformations of the system when the loads became heavier. Experimental results showed that for the loads we applied to the system, even with noticeable deformations, our model is robust. We will work in the future to improve the design of the Strong Arm to decrease the deformations when loads increase. To further address this issue, we could make adjustments to the stability zone of the model output by decreasing the footprint of the EPW used in the model. Lastly, in this article we did not consider possible disturbances during the transfer, for example, exerting forces from accidentally touching the Strong Arm during transfers. In the future, when we develop better control systems, this factor should be considered when deciding the parameters for the control.

\section{CONCLUSIONS}

In this article, we investigated the tip-over stability analysis of an EPW-mounted robotic transfer device (Strong Arm) system. A simple tip-over stability analysis was introduced to gain a basic understanding of the nature of the problem. A mathematical model of the Strong Arm and EPW-based prediction model to determine the COM distribution of the system was described. The prediction model is composed of only the most fundamental structures
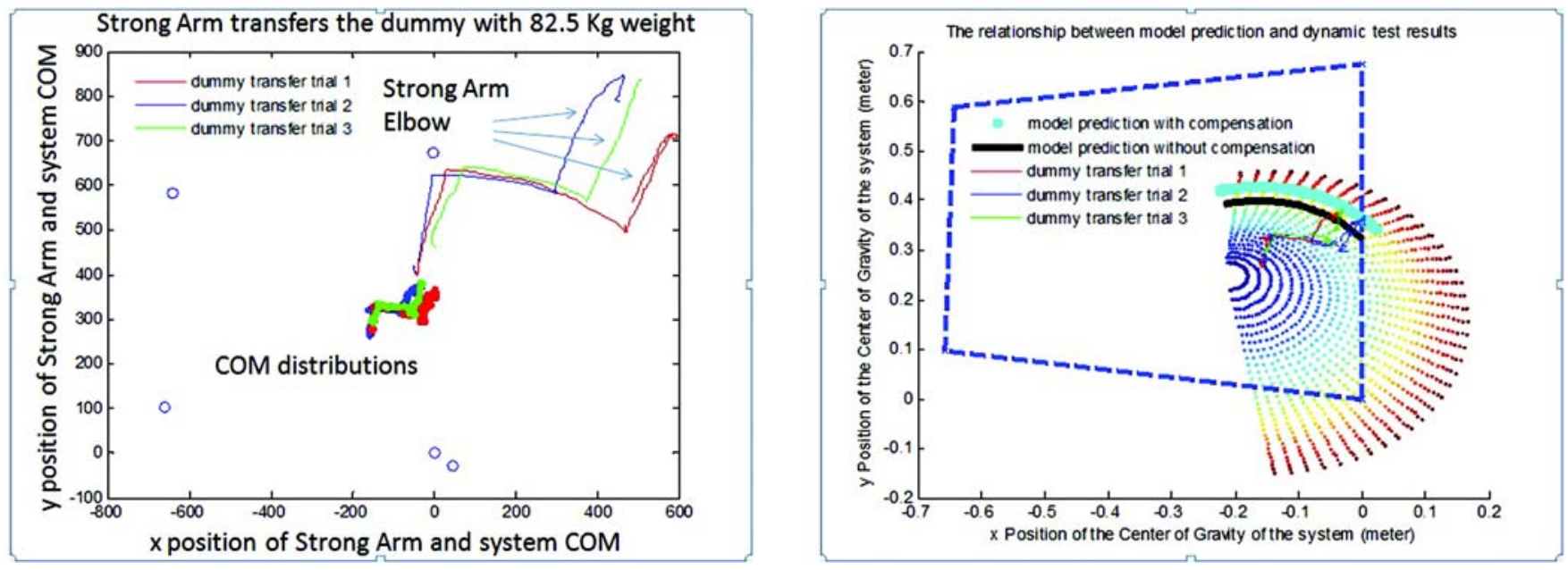

Figure 10.

Center of mass (COM) distribution for four different angles with variance loads on Strong Arm. 
and was developed based on the loads, payload position (rotation angle and length of the payload from the rotation axis), and arm position (carriage position on the track system). The COM distribution regarding the footprint of the EPW provides a useful index that characterizes the system's tendency to tip over for any given system configuration. The system is considered stable as long as the COM remains inside the footprint of the EPW.

Based on the model, the static stability analysis was performed while the EPW was stationary. The maximum possible payload calculated for the Strong Arm being rotated the whole range around the EPW was $60 \mathrm{~kg}$. The model reveals the general nature of the system's tip-over stability. The system is found to be the least stable when the Strong Arm is fully extended directly pointed to the front or to the sides. Moreover, for each known weight, the model predicts the range of motion the Strong Arm could be operated; for example, for a payload of $90 \mathrm{~kg}$, the fully extended Strong Arm could be operated stably at the $0^{\circ}$ to $45^{\circ}$ zone. The model was then verified with experimental tests. From the test results, we found that the model performance was consistent for different test conditions (with different loads and different rotation angles).

The effects of changing key Strong Arm parameters on tip-over stability were also analyzed and verified. The system's stability is reduced when the Strong Arm is extended and carrying a heavier payload. The system's stability is reduced when the carriage moves forward when the Strong Arm is pointed forward with a heavier payload. All the analysis results agreed between the model and experimental results.

The dynamic performances of the system were evaluated by comparing the COM distribution of the model prediction and the COM distribution measures directly from the experiments. Although the model predicted the COM distribution when the Strong Arm is moved slowly, the model did not predict the end of the transfer well due to inertia. Therefore, either a dynamic model should be developed to analyze the stability or a well-developed transfer protocol should be developed to control the inertia effect during transfers.

Three loads close to human weight were selected and transferred by the Strong Arm. As shown in Figures 8 to 10, the Strong Arm transferred all three weights successfully without tip-over. In addition, with the heavier weights applied, the inertia effect caused by the dynamic movement decreased compared with lower weights. This is expected since, as the weight increases and the Strong
Arm moves slowly, there is less free body movement due to the heavier mass. There were noticeable deformations of the system when the loads reached more than $50 \mathrm{~kg}$, and the model was affected because the deformation leads to the change of the distance between loads and carriage. To solve the problem, a linear compensator was added based on the relationship between deformationcaused length changes and weight applied. From the three loads tested, the compensator worked very well and the corrected model with the compensator could successfully predict the COM distributions. However, this compensator might not be robust due to the nonlinear nature of the deformation. In the future, either we will modify the design to control the deformations or we will need to develop a nonlinear compensator for the model to accurately predict the COM distribution.

In summary, this article develops a prediction model for the stability of an EPW-mounted robotic transfer device. The model prediction results were verified by different experimental conditions to demonstrate its sensitivity and robustness. The Strong Arm could successfully transfer up to $83.25 \mathrm{~kg}$ for current transfer protocol. Based on the model and experimental verification, we are working on implementing the model to the control of the Strong Arm. It is called manifold control, which will define the safe operation zone of the Strong Arm. In addition, before each transfer, a self-calibration will be conducted to calculate the safe zone based on user weight and initial position of the Strong Arm.

\section{ACKNOWLEDGMENTS}

\section{Author Contributions:}

Study concept and design: H. Wang, G. G. Grindle, R. A. Cooper. Acquisition of data: H. Wang, C. Tsai, H. Jeannis, A. Kelleher. Analysis and interpretation of data: H. Wang, C. Chung, C. Tsai. Drafting of manuscript: $\mathrm{H}$. Wang.

Critical revision of manuscript for important intellectual content: H. Wang, G. G. Grindle, R. A. Cooper.

Obtained funding: H. Wang, G. G. Grindle, R. A. Cooper, A. Kelleher. Administrative, technical, or material support: A. Kelleher.

Study supervision: R. A. Cooper.

Financial Disclosures: The authors have declared that no competing interests exist.

Funding/Support: This material was based on work supported in part by the Quality of Life Technology Engineering Research Center, National Science Foundation (grant ERC-0540865), and Department of Veterans Affairs Rehabilitation Research and Development Service (grant B3142C). 
Additional Contributions: The authors would like to thank Michael Lain at the Human Engineering Research Laboratories for his valuable help on this work.

Disclaimer: The contents of this article do not represent the views of the Department of Veterans Affairs or the U.S. Government.

\section{REFERENCES}

1. Koontz A, Toro M, Kankipati P, Naber M, Cooper R. An expert review of the scientific literature on independent wheelchair transfers. Disabil Rehabil Assist Technol. 2012;7(1):20-29. [PMID:21591884] http://dx.doi.org/10.3109/17483107.2011.553983

2. Kirby RL, Smith C. Fall during a wheelchair transfer: A case of mismatched brakes. Am J Phys Med Rehabil. 2001; 80(4):302-4. [PMID:11277138] http://dx.doi.org/10.1097/00002060-200104000-00015

3. Ummat S, Kirby RL. Nonfatal wheelchair-related accidents reported to the National Electronic Injury Surveillance System. Am J Phys Med Rehabil. 1994;73(3):163-67.

[PMID:8198772]

http://dx.doi.org/10.1097/00002060-199406000-00004

4. Bostelman R, Albus J. Survey of patient mobility and lift technologies: Toward advancements and standards [Internet]. Cite SeerX. University Park (PA): College of Information Sciences and Technology, Pennsylvania State University; 2006. Available from: http://citeseerx.ist.psu.edu/viewdoc/ summary?doi=10.1.1.108.1207

5. Hess JA, Kincl LD, Mandeville DS. Comparison of three singleperson manual patient techniques for bed-to-wheelchair transfers. Home Healthc Nurse. 2007;25(9):577-79.

[PMID:18049253] http://dx.doi.org/10.1097/01.NHH.0000296114.33696.e5

6. Manufacturing metrology and standards for the Health Care Enterprise Program Healthcare Mobility Project [Internet]. Gaithersburg (MD): National Institute of Standards and Technology; 2006. Available from: http://www.nist.gov/el/isd/healthcaremobility.cfm

7. Miller A, Engst C, Tate RB, Yassi A. Evaluation of the effectiveness of portable ceiling lifts in a new long-term care facility. Appl Ergon. 2006;37(3):377-85.

[PMID:16380072]

http://dx.doi.org/10.1016/j.apergo.2005.05.012

8. Panasonic's future outlook on robotics \& health care [Internet]. Plastic Pals; 2009. Available from: http://www.plasticpals. $\mathrm{com} / \mathrm{p}=15775$

9. Bostelman R, Albus J, Chang T, Hong T, Agrawal S, Ryu J. HLPR chair: A novel indoor mobility-assist and lift system. Proceedings of the ASME 2007 International Engineering Technical Conferences and Computers and
Information in Engineering Conference; 2007 Sep 4-7; Las Vegas, NV. http://dx.doi.org/10.1115/DETC2007-35295

10. Bostelman R, Albus J. Robotic patient transfer and rehabilitation device for patient care facilities or the home. Adv Robot. 2008;22(12):1287-1307. http://dx.doi.org/10.1163/156855308X344837

11. Bostelman R, Ryu JC, Chang T, Johnson J, Agrawal SK. An advanced patient lift and transfer device for the home. J Med Device. 2010;4(1):1-8.

12. Cooper RA, Grindle GG, Vazquez JJ, Xu J, Wang H, Candiotti J, Salatin B, Houston E, Kelleher AR, Cooper RM, Teodorski E, Beach S. Personal mobility and manipulation appliance-Design, development, and initial testing. Proc IEEE Inst Electr Electron Eng. 2012;100(8):2505-11. http://dx.doi.org/10.1109/JPROC.2012.2200537

13. Jeannis H, Grindle GG, Kelleher A, Wang H, Brewer B, Cooper RA. Initial development of direct interaction for a transfer robotic arm system for caregivers. Proceedings of the 2013 IEEE International Conference on Rehabilitation Robotics; 2013 Jun 24-26; Seattle, WA. p. 1-5.

14. Papadopoulos E, Rey D. A new measure of tipover stability margin for mobile manipulators. Proceedings of the 1996 IEEE International Conference on Robotics and Automation; 1996 Apr 22-28; Minneapolis, MN. p. 3111-16.

15. Churchill E, Laubach LL, McConville JT, Tebbetts I. Anthropometric source book. Volume 1: Anthropometry for designers. Washington (DC): NASA; 1978. p. 7-78.

16. ST-E-1321 Japanese female body size. National Space Development Agency of Japan, 06-05-86.

17. Halls SB, Hanson J. Average height and weight charts [Internet]. Halls.md; 2000 [updated 2008 May 26]. Available from: http://www.halls.md/chart/height-weight.htm

18. Koontz AM, Kankipati P, Lin YS, Cooper RA, Boninger ML. Upper limb kinetic analysis of three sitting pivot wheelchair transfer techniques. Clin Biomech (Bristol, Avon). 2011;26(9):923-29. [PMID:21664733] http://dx.doi.org/10.1016/j.clinbiomech.2011.05.005

Submitted for publication November 7, 2013. Accepted in revised form December 30, 2013.

This article and any supplementary material should be cited as follows:

Wang H, Tsai C, Jeannis H, Chung C, Kelleher A, Grindle GG, Cooper RA. Stability analysis of electrical powered wheelchair-mounted robotic-assisted transfer device. J Rehabil Res Dev. 2014;51(5):761-74. http://dx.doi.org/10.1682/JRRD.2013.11.0240

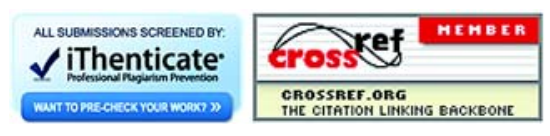


